\title{
A UTILIZAÇÃO DE AMBIENTES VIRTUAIS NO ENSINO DE BIOQUIMICA. UM ESTUDO DE CASO NA UNICSUL.
}

\author{
Carmem Lúcia Costa Amaral*, Rubens César Lopes Figueira e Marcelo Paes de Barros \\ Centro de Ciências Biológicas e da Saúde, Universidade Cruzeiro do Sul, UNICSUL. \\ *carmem.amaral@unicsul.br
}

\section{Resumo:}

A Universidade Cruzeiro do Sul (UNICSUL) vem aplicando nesses dois últimos anos uma nova estratégia de ensino, disponibilizando conteúdo didático de bioquímica em sua rede interna de computadores como suporte ao ensino tradicional em sala de aula. Essa estratégia utiliza o Black Board Learning System, um software desenvolvido para ser utilizado por profissionais interessados em aplicar novas tecnologias interativas no processo de ensino-aprendizagem por meio de um sistema de gestão de conteúdos e comunicação online. Este recurso vem sendo disponibilizado na disciplina de bioquímica desde o ano de 2003. A estratégia consistiu em lançar com antecipação de uma semana a teoria que seria estudada em sala de aula a partir de listas de exercícios e estudos de casos que seriam posteriormente analisados em grupos. A avaliação do uso desta ferramenta no processo ensino-aprendizagem foi realizada comparando-se o índice de aprovação e o desempenho do aluno nesta disciplina nos dois últimos anos com os anos anteriores (antes da utilização da metodologia).

Os resultados observados mostram uma maior interação dos alunos com a disciplina em sala de aula e um decréscimo no índice de reprovação e evasão nesta disciplina em relação aos anos anteriores para os mesmos professores.

\begin{abstract}
:
In the last two years the UNICSUL following a new institutional proposal of "Practical Learning", has introduced new teaching strategies into several graduating courses, mostly based on the University's intranet web. One of these approaches uses the Black Board Learning System, a ready-to-use and uncomplicated system that allows students to freely access specific topics of several disciplines (including the course schedule, practicing exercises and supporting extra activities) and also communicate online with colleagues and teachers. The software is provided with sufficient resources to improve classroom community sense launched by the emphasis on the general interactive skills in the teaching-learning process. This work focuses on the application of the Black Board system in the Biochemistry course for Nursery graduating students at UNICSUL during 18 months. Partial results points out significant positive achievements of the Black Board-experienced students based on the higher approval indexes compared to traditionally taught students (before 2003).
\end{abstract}




\section{Introdução}

Nos últimos anos tem-se observado em boa parte das instituições de ensino superior uma grande dificuldade dos alunos ingressantes nos cursos da área de saúde na aprendizagem de tópicos da disciplina de Bioquímica. Este problema apresentado pelos estudantes deve-se principalmente ao fato desta disciplina utilizar muito da abstração para descrever os fenômenos que acontecem em nível molecular, além de exigir conhecimentos básicos de química, independente do curso a que se destina. A realidade educacional brasileira tem demonstrado que às instituições privadas de ensino superior têm aportado principalmente alunos que se caracterizam por serem oriundos das classes populares e por terem realizado a formação básica na rede pública. ${ }^{1}$ Ao se levar em conta a precariedade da qualidade dessa rede de ensino e em função da complexidade dos conteúdos de bioquímica, formas tradicionais de ensino pode tornar o aprendizado ainda mais difícil quando tratado apenas com o auxílio de lousa e retroprojetor. Além disso, as mudanças ocorridas nos últimos anos, sobretudo no que diz respeito às novas tecnologias, apontam o descompasso das formas tradicionais de ensino frente às novas linguagens que o aluno se depara no seu dia-a-dia. Essas transformações também modificam a maneira de se apreender o mundo e construir o conhecimento. Viana e Araújo ${ }^{2}$, seguindo os estudos de Pierre e Levy, destacam que o reconhecimento da presença das novas tecnologias nos leva a repensar as metodologias aplicadas ao ensino e a buscar outras formas de construir o conhecimento.

$\mathrm{Na}$ elaboração do projeto pedagógico, a universidade deve levar em consideração essa realidade, bem como a expectativa do aluno e a necessidade de completar sua preparação para adequá-lo às exigências do ensino superior. Com essa preocupação, a Universidade Cruzeiro do Sul (UNICSUL) vem implementado uma nova metodologia institucional em que o aluno deve apropriar-se de um novo conceito de aprendizagem, que rompe com o modelo transmissivo-reprodutivo, a que esteve submetido ao longo de toda sua escolaridade.

Para o sucesso dessa nova metodologia a UNICSUL vem estimulando o seu corpo docente a desenvolver novas estratégias de ensino, que possam contribuir para a motivação e envolvimento dos alunos, ao mesmo tempo em que permite ao professor uma atuação mais interativa. O objetivo desse procedimento é modificar a visão do professor e do aluno de que a sala de aula se constitui em um espaço físico onde o professor transmite seus conhecimentos e experiências, cabendo aos alunos simplesmente a tarefa de "copiar a matéria", ouvir, às vezes elaborar perguntas e, na maioria das vezes, reproduzir (quando cobrado) as informações que o professor ensinou. Esta nova metodologia se traduz por aprender na prática, exigindo uma maior participação dos alunos, isto é, o aluno passa de mero expectador para agente em seu processo de aprendizagem. Segundo este novo ponto de vista, uma das maneiras de modificar esse quadro é entender que tanto os alunos quanto o professor se encontram na sala de aula para juntos realizarem uma série de ações como estudar, ler, discutir e debater, solucionar dúvidas e trocar informações a respeito do conteúdo da disciplina. Para a realização desta tarefa de modo mais eficiente, o professor deve procurar aplicar novas metodologias e estratégias de ensino. . $^{3-4}$

Tendo em vista essa preocupação, a UNICSUL, vem estimulando seus docentes a conhecer ambientes educacionais especialmente projetados para a disponibilização de conteúdo didático para suporte aos alunos. ${ }^{5,6}$ Dentre eles, o Black Board Learning System que é um dos ambientes de aprendizagem que vem sendo bastante utilizado, por um grande número de professores, pois foi projetado para propiciar uma grande alteração nas formas de apresentação de conteúdos e, também, uma maior interação entre alunos e professores, com o objetivo de melhorar a compreensão do conteúdo das disciplinas.

Como proposta inicial, escolheu-se trabalhar com esta nova estratégia nos cursos de Enfermagem, para a disciplina de Bioquímica. Os dados levantados, nestes 
últimos dois anos, confirmam os resultados, pois constatou-se uma maior interação entre alunos e professores, assim como uma melhora na compreensão do conteúdo da disciplina.

O interesse, por este ambiente, se deve ao fato de o mesmo permitir a disponibilização de material didático e fóruns online, com painel de discussão e sala de bate-papo, os quais dispõem de áreas para a publicação de opiniões e, desta forma, cada estudante pode ler as respostas dos colegas e discuti-las entre si. Acredita-se que isto beneficia os estudantes uma vez que os habilita, assim, a combinar novas opiniões com suas próprias e por meio da discussão desenvolver uma base sólida de aprendizado.

Para isto, deve-se, entretanto, ter em mente que nenhum programa de informática funciona automaticamente como desencadeador do processo de aprendizagem, ou seja, o sucesso de um software educacional em promover a aprendizagem depende de sua integração ao currículo e às atividades da sala de aula, bem como do nível de interação entre aluno-aluno, aluno-professor e a interatividade com o computador. ${ }^{4,7-8}$

Levando em conta os benefícios oferecidos por este ambiente virtual e as dificuldades encontradas em sala de aula, resolveu-se experimentar uma nova forma de desenvolver nossos conteúdos, visando uma maior interação entre os discentes e docentes que participam do processo e, conseqüentemente, uma aprendizagem mais eficaz.

\section{Metodologia}

Durante os dois últimos anos, foram disponibilizados semanalmente na rede interna de computadores da UNICSUL os conteúdos da disciplina de Bioquímica no ambiente virtual de aprendizagem. Este material não era apenas composto pelo conteúdo básico, mas também com apresentação e discussão de casos clínicos referentes aos tópicos em questão, listas de exercícios para garantir uma melhor fixação do conteúdo apresentados em sala de aula e links para melhor visualização de estruturas moleculares e vias metabólicas. Essa estratégia foi utilizada no curso de Enfermagem, com o auxílio do Núcleo de Educação à Distância ligado ao Centro de Ciências Exatas e Tecnológicas (CETEC) da Universidade.

\section{Resultados e Discussão}

Antes de se utilizar esta metodologia de ensino-aprendizagem a seus alunos, a UNICSUL realizou no ano de 2002, por meio da Comissão Permanente de Avaliação Institucional (CPAI), uma pesquisa que teve como objetivo adquirir conhecimento do nível de acesso dos alunos à internet e sua freqüência de utilização nos campi da Universidade. Os resultados desta pesquisa estão apresentados no Gráfico 1 e no Gráfico 2. 


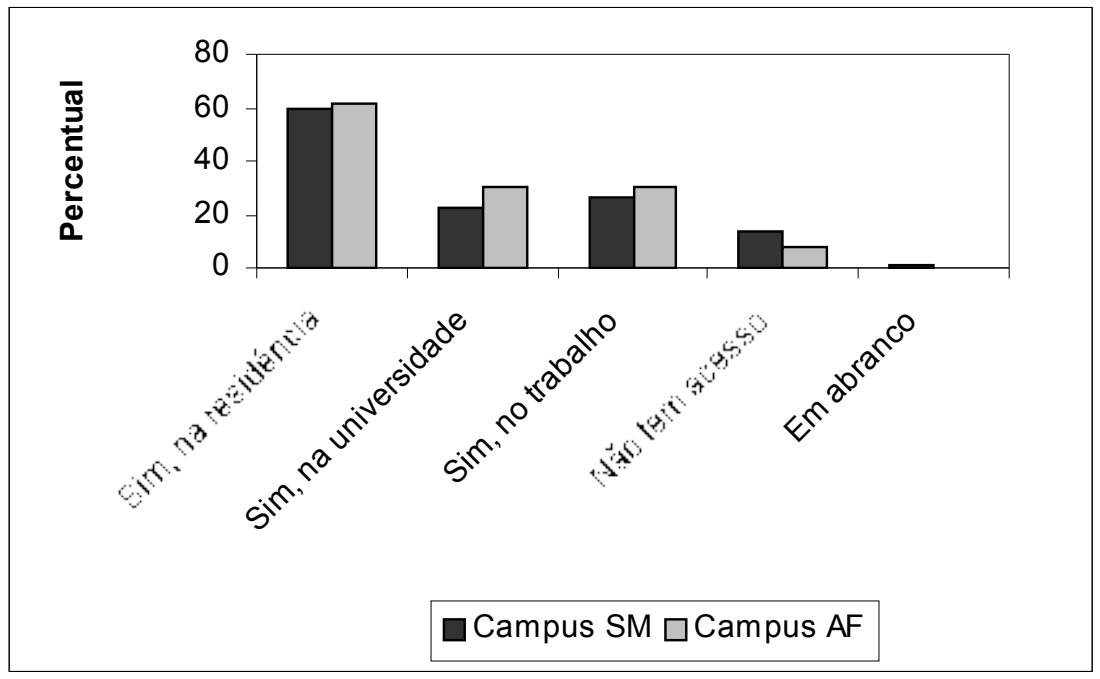

GRÁFICO 1 - Percentual de alunos que utilizam a internet nos Campi da UNICSUL (ano 2002). SM = São Miguel. AF = Anália Franco.

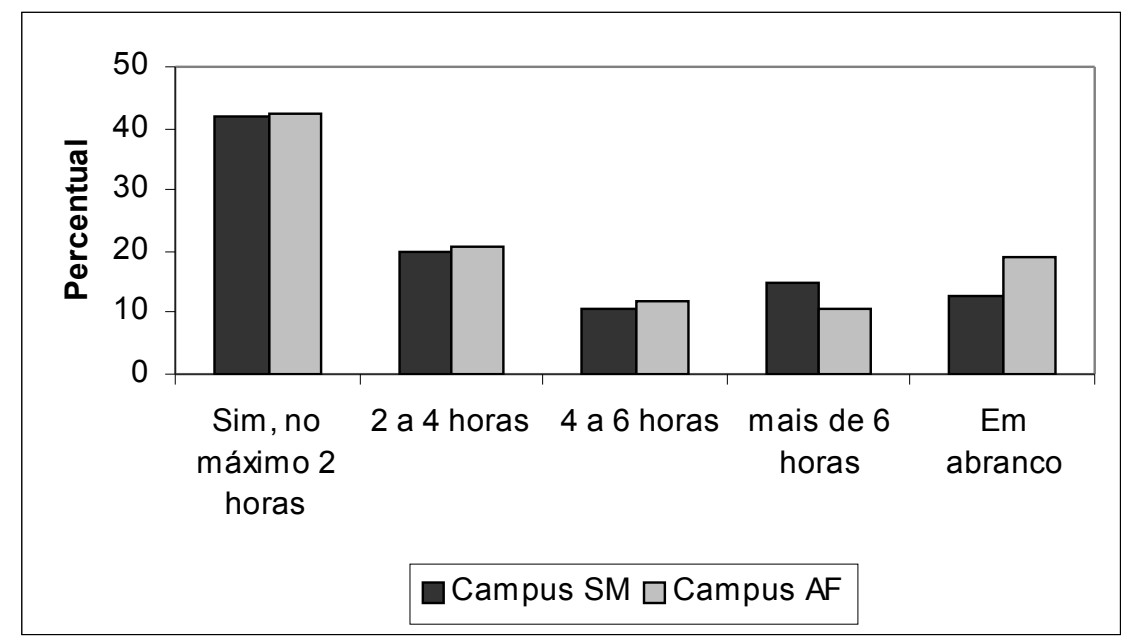

GRÁFICO 2 - Número de horas semanais de utilização da Internet pelos alunos da UNICSUL (ano 2002). SM = São Miguel. AF =Anália Franco.

No Gráfico 1 pode-se observar que em 2003, aproximadamente $60 \%$ dos estudantes tinham acesso à internet em suas residências e um número significativo tinha acesso por meio da utilização do Laboratório de Informática da Universidade e no trabalho. Apenas cerca de $10 \%$ dos alunos não possuíam acesso a internet, muito embora a universidade ofereça laboratório de informática. No Gráfico 2 nota-se que a incidência maior de utilização deste recurso era de duas horas semanais, embora nas demais horas o índice fosse representativo. Estes resultados indicaram a existência do hábito, ao menos, em um nível razoável, de utilização do recurso da internet por parte do corpo discente da instituição. Com base neste resultado, constatou-se que o computador fazia parte do cotidiano dos estudantes, indicando que poderia também ser utilizado como mais uma ferramenta no processo de ensino-aprendizagem, somando-se às demais e enriquecendo o processo.

Os dados apresentados representam um levantamento feito entre todos os cursos da Universidade. Contudo, ao se observar apenas o curso de Enfermagem, 0 percentual praticamente se manteve, mostrando que o uso de tecnologia poderia ser aplicado.

Após essa pesquisa, o Centro de Ciências Biológicas e da Saúde juntamente com seus professores e coordenadores, decidiram aplicar essa nova estratégia à disciplina de Bioquímica, uma vez que esta constitui uma disciplina básica da grade 
curricular de vários cursos da área de Ciências Biológicas/Biomédica. Outro aspecto que influenciou na utilização desta metodologia foi o alto grau de dificuldade apresentado pelos alunos nos anos anteriores, bem como o alto percentual de reprovação. Boa parte da dificuldade, como citado anteriormente, deve-se a abstração para a visualização espacial das estruturas químicas das principais biomoléculas, bem como as transformações químicas envolvidas nas respectivas vias metabólicas. Conhecendo-se essas dificuldades, os conteúdos e as atividades da disciplina foram estruturados de modo a fornecer aos alunos não só teoria e lista de exercícios, mas, também um ambiente de discussão de dúvidas na sala de bate papo, além da apresentação de casos clínicos que seriam posteriormente discutidos em sala de aula.

Esse ambiente de aprendizagem foi apresentado aos alunos no começo do primeiro semestre de 2003 e 2004, de modo que todos conhecessem como seria desenvolvida a disciplina durante todo o ano letivo. Sua avaliação no processo ensinoaprendizagem foi realizada comparando-se o índice de aprovação nos anos citados acima com os anos de 2001 e 2002 (antes da utilização da metodologia). Para fazer com que todos os alunos estivessem sincronizados no conteúdo a ser discutido em sala de aula e evitar que alguns se adiantassem, esses eram disponibilizados gradualmente, um a cada semana. Algumas atividades propostas, como por exemplo, as listas de exercícios, tinham um prazo de entrega para fins de avaliação. A discussão de casos clínicos era realizada em sala de aula. Em alguns momentos os alunos liam o material e faziam um resumo que depois era discutido em sala de aula.

Os resultados da avaliação no índice de aprovação estão apresentados na Tabela 1. Nesta Tabela os números em parênteses significam total de alunos que utilizaram o ambiente no processo de ensino-aprendizagem com suas respectivas percentagem de aprovação. O número de alunos representa a soma de duas turmas do período diurno e uma do período noturno.

TABELA 1 - Índice de Aprovação em Bioquímica para o curso de Enfermagem na UNICSUL. Os números em parênteses significam alunos que utilizaram o ambiente virtual de aprendizagem.

\begin{tabular}{|c|c|c|c|}
\hline ANO & $\begin{array}{c}\text { No TOTAL } \\
\text { ALUNOS }\end{array}$ & $\mathbf{N}^{0}$ APROVADOS & \% APROVAÇÃO \\
\hline 2001 & 160 & 95 & 59 \\
\hline 2002 & 178 & 108 & 60 \\
\hline 2003 & $202(150)$ & $147(122)$ & $17(83)$ \\
\hline 2004 & $245(190)$ & $185(155)$ & $16,2(83,8)$ \\
\hline
\end{tabular}

Nota-se na Tabela 1 que nos anos de 2003 e 2004, período no qual os alunos tiveram acesso ao ambiente, houve um significativo aumento no índice de aprovação, fato que sugere uma maior absorção dos conceitos e, possivelmente, uma maior compreensão do contexto em que se insere a disciplina por parte dos alunos. Observou-se, paralelamente, que as aulas em classe ganharam um novo componente: o objetivo dos estudantes em esclarecer as dúvidas derivadas do material disponibilizado, levando esses alunos a terem uma maior participação em sala de 
aula. Esse resultado foi atribuído ao contato prévio do aluno com o conhecimento disponibilizado no ambiente. Acredita-se que uma das vantagens desta estratégia seja a de permitir ao aluno programar seus momentos de estudo, aprendendo assim a aprender.

\section{CONCLUSÃO}

As estratégias de ensino constituem-se em instrumentos que têm por objetivo auxiliar o professor no desenvolvimento do processo ensino-aprendizagem. Seu uso implica numa seleção intencional e diretiva daquelas que melhor funcionam como mediadoras e facilitadoras deste processo. Há uma variedade de estratégias de ensino à disposição do docente, se fazendo necessárias à análise, seleção, readequação e adaptação do método de acordo com o paradigma de educação. Tal adaptação, por sua vez, envolve a concepção de ensino-aprendizagem do profissional, sua concepção metodológica, o conteúdo a ser trabalhado, os objetivos de ensino a serem alcançados, as características da turma, o número de alunos em sala, as condições de infra-estrutura e materiais didáticos disponíveis e as suas habilidades pessoais, razão pela qual é importante que o docente estude e analise as possibilidades inerentes às estratégias de ensino a ser aplicada. Tendo-se como parâmetro essa análise na escolha da estratégia e com base nos resultados obtidos nestes dois últimos anos da utilização de ambiente virtual de aprendizagem no estudo de Bioquímica na UNICSUL, pode-se concluir que a estratégia de ensino escolhida neste trabalho mostrou-se eficaz no processo ensino-aprendizagem.

Embora o ambiente virtual utilizado seja apenas uma ferramenta (e não a finalidade em si) na exploração do uso das tecnologias de informação e comunicação na educação, ele, indubitavelmente, permite a adaptação para diversas abordagens e estratégias de uso ${ }^{6,11}$. É importante salientar que essa nova estratégia exige um trabalho muito maior do aluno, pois ele está sendo avaliado progressivamente, uma vez que cada aula é considerada como uma nova etapa do processo, não podendo ser rompida. Os alunos, habituados com as aulas tradicionais, colocam alguma resistência ao novo processo, pois o mesmo exige que ele avalie sua aprendizagem.

\section{Referências Bibliográficas}

${ }^{[1]}$ MACEDO, I.F.G e CATARDO, L. A M. (2001) Projeto pedagógico de cursos de graduação: a construção em debate. Revista UNICSUL, 6 (8), 131-140.

${ }^{[2]}$ VIANNA, D.M.; ARAUJO, R.S. (2004)., Buscando elementos na Internet para uma nova Proposta Pedagógica. In CARVALHO, A.M.P., Ensino de Ciências. Unindo a Pesquisa e a Prática. São Paulo: Thomson, pp.135-151.

[3] MASSETO, M.T., (2003) Docência Universitária: Repensando a Aula IN: Teodoro, A. e Vasconcelos, M. L., Ensinar e Aprender no Ensino Superior, São Paulo: Mackenzie e Cortez, pp. 79-108.

${ }^{[4]}$ ABREU, M.C. e MASSETO, M.T., O Professor Universitário em Aula, 8a Edição, São Paulo: MG Ed. Associados, 1990, pp., 47

${ }^{[5]}$ ARAÚJO JR., C. F.; OLIVEIRA, I. C. A; AMARAL, L.H.; SILVEIRA, I. F. (2003) Novas Tecnologias de Informação e Comunicação e Educação a Distância no Ensino Superior: experiências na área de Computação e Informática. In: OEB 2003 - Online 
Educa Barcelona, Barcelona. Proceedings do OEB 2003 - Online Educa Barcelona. Berlin: ICWE GmbH, 2003. pp. $209-213$

${ }^{[6]}$ ARAUJO JR., C. F.; NAITO, L.; AMARAL, L.H.; TURINE, M.A.S. (1999) Metodologia para Seleção de Tecnologias para Educação a Distância Mediada por Computador (EDMC), In: WISE99- Workshop Internacional Sobre Educação Virtual, Fortaleza, Ceará. pp. 266-275.

${ }^{[7]}$ ALEXANDER, S., Teaching and Learning on the World Wide Web. AusWeb 97 Conference. 1997. http://ausweb.scu.edu.au/.

${ }^{[8]}$ CARRAHER, D.W., O Papel do Computador na Aprendizagem. Acesso, 3 (5): 19-21, 1992.

${ }^{[9]}$ GALVIS, A.H. Usos educativos de computadores: possibilidades y requerimentos. Revista de Tecnología Educativa (Santiago - Chile) 9: 261-275, 1986.

${ }^{[10]}$ LOLLINI, P. Didática e Computadores - Quando e como a informática na escola. São Paulo: Edições Loyola. 1991

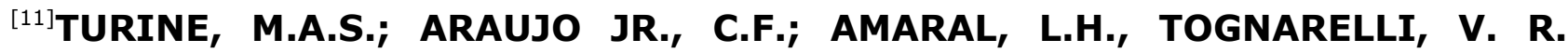
Análise dos Resultados da Avaliação Institucional para a Melhoria da Qualidade dos Cursos de Computação e Informática. To appear in IEEE - ICECE2000 International Conference on Engineering and Computer Education, Brazil, São Paulo, Agosto de 2000, homepage: http://www.sp.senac.br/icece2000 